\title{
LOCAL RESOLVENTS OF OPERATORS WITH ONE-DIMENSIONAL SELF-COMMUTATOR
}

\author{
CONSTANTIN APOSTOL AND KEVIN CLANCEY
}

\begin{abstract}
Let $T=H+i J$ be an irreducible operator on a Hilbert space with one-dimensional self-commutator. It is known that the selfadjoint operator $H$ is absolutely continuous. Let $E_{H}$ denote the absolutely continuous support of $H$. In this note the following theorem is proven:

THEOREM. If there exists a real number $p$ such that ess inf $E_{H}<p$ $<\operatorname{ess} \sup E_{H}$ and $\int_{E_{H}}|t-p|^{-1} d t<\infty$, then the operator $T$ has a nontrivial invariant subspace.
\end{abstract}

Let $\mathcal{H}$ be a separable Hilbert space with inner product ( , ). Let $T$ be a bounded linear operator on $\mathcal{H}$. The operator $T$ is called hyponormal in case its self-commutator $T^{*} T-T T^{*}=D$ is nonnegative. If the adjoint $T^{*}$ is a hyponormal operator, then $T$ is called cohyponormal. If the self-commutator $T^{*} T-T T^{*}$ is a one-dimensional operator, then either $T$ or $T^{*}$ is hyponormal.

It is not known at present whether every operator with a one-dimensional self-commutator has a nontrivial invariant subspace. In this note a result is described which increases the class of operators with one-dimensional selfcommutator that are known to have nontrivial invariant subspaces.

Let $T$ be a cohyponormal operator. The cartesian decomposition of $T$ will be expressed $T=H+i J$, where $H$ and $J$ are selfadjoint. For the purposes of this note it can be assumed that $T$ is an irreducible operator. In this case the selfadjoint operators $H$ and $J$ are absolutely continuous [10, Theorem 3.2.1]. Suppose $H=\int t d G_{t}$ is the spectral resolution of $H$. Then there is a Borel set $E_{H}$ in the real line, determined up to a set of measure zero with the property $\int_{E_{H}} d G_{t}=I$ and if $\int_{F} d G_{t}=I$, for $F \subset E_{H}$, then $E_{H} \backslash F$ is of Lebesgue measure zero. This set $E_{H}$ (really $E_{H}$ is an equivalence class of Borel sets) will be called the absolutely continuous support of $H$.

It is known [10, Theorem 3.4.1] that if $T=H+i J$ is cohyponormal, then $\sigma(H)$ (the spectrum of $H$ ) is the projection on the $x$-axis of $\sigma(T)$. Similarly, $\sigma(J)$ is the projection of $\sigma(T)$ onto the $y$-axis. An operator with disconnected spectrum is known to have nontrivial invariant subspaces. It follows that every cohyponormal operator $T=H+i J$, where $\sigma(H)$ or $\sigma(J)$ is not an interval, has a nontrivial invariant subspace.

The main result in this note is the following:

THEOREM 1. Let $T=H+i J$ be an irreducible operator with one-dimensional self-commutator. Let $E_{H}$ be the absolutely continuous support of $H$ and assume

Received by the editors December 13, 1974 and, in revised form, February 19, 1975.

AMS (MOS) subject classifications (1970). Primary 47B20; Secondary 47A15. 
$\sigma(H)=[a, b]$. If for some $p \in(a, b), \int_{E_{H}}|t-p|^{-1} d t<\infty$, then the operator $T$ has a nontrivial invariant subspace.

The proof of Theorem 1 will be based on some estimates of the local resolvent of the vector in the range of $T^{*} T-T T^{*}$. These estimates are deduced from results in Carey and Pincus [1]. The fact that local resolvents can be used to obtain invariant subspaces for hyponormal operators is in Stampfli [12]. In the case where $T$ is cohyponormal with no point spectrum $T T^{*}-T^{*} T=\langle, \varphi\rangle \varphi$, then there is a weakly continuous extension of $(T-\lambda)^{-1} \varphi$ onto the whole complex plane C (see Putnam [11]). In the final section of this note it is shown that this extension of $(T-\lambda)^{-1} \varphi$ is not an analytic extension across the boundary of $\sigma(T)$.

1. Preliminaries and notations. From now on it will be assumed that $T=H+i J$ is an irreducible cohyponormal operator with one-dimensional self-commutator. The notation $T_{\lambda}$ will be used for $T-\lambda I$. It is convenient to normalize $T$ such that $T T^{*}-T^{*} T=(2 / \pi)(, \varphi) \varphi$, where $\varphi$ is a unit vector in Fo.

J. D. Pincus [7] has provided a useful unitary invariant for the operator $T$. There is a bounded measurable function $g$ defined on the plane which satisfies

$$
\begin{aligned}
1+(\pi i)^{-1}\left((J-\omega)^{-1}(H-z)^{-1} \varphi, \varphi\right) \\
\quad=\exp \left\{\frac{1}{2 \pi i} \iint g(x+i y) \frac{d x d y}{(x-z)(y-\omega)}\right\},
\end{aligned}
$$

where $\operatorname{Im} z \neq 0$ and $\operatorname{Im} \omega \neq 0$. The function $g$ is called the principal funcion of $T$ and has the following properties:

(i) The function $g$ vanishes off $E_{H} \times E_{J}$ and satisfies $0 \leqslant g \leqslant 1$ (see [1]).

(ii) The spectrum of $T$ consists of all complex $\lambda$ such that every neighborhood of $\lambda$ intersects the set $\{z: g(z) \neq 0\}$ in a set of positive measure (see [4] and [8]).

(iii) The essential spectrum of $T$ consists of all complex $\lambda$ such that every neighborhood of $\lambda$ intersects the sets $\{z: g(z) \neq 0\}$ and $\{z: g(z) \neq 1\}$ in a set of positive measure [1].

(iv) The point spectrum of $T$ consists of all complex $\lambda=\mu+i v$ such that

$$
\iint_{B(\lambda)} \frac{1-g(x+i y)}{(x-\mu)^{2}+(y-\nu)^{2}} d x d y<\infty ;
$$

here, $B(\lambda)=\{z:|z-\lambda|<1\}$, [1].

Let $\lambda=\mu+i \nu$ be a complex number. It is useful to introduce the function

$$
\nu_{g}(\lambda)=\frac{1}{\pi} \iint \frac{g(x+i y)}{(x-\mu)^{2}+(y-\nu)^{2}} d x d y .
$$

For our purposes we will need the following estimate which follows easily from the results in [1] (see, in particular the proof of Theorem 6 of [1]).

(v) Let $\lambda$ be complex and let $T_{\lambda}^{*} T_{\lambda}=\int t d E_{t}, T_{\lambda} T_{\lambda}^{*}=\int t d F_{t}$ be the spectral resolutions of $T_{\lambda}^{*} T_{\lambda}$ and $T_{\lambda} T_{\lambda}^{*}$, respectively. Then 


$$
\begin{aligned}
1+\frac{2}{\pi} \int t^{-1} d\left(E_{t} \varphi, \varphi\right) & =\exp \left[\nu_{g}(\lambda)\right], \\
1-\frac{2}{\pi} \int t^{-1} d\left(F_{t} \varphi, \varphi\right) & =\exp \left[-\nu_{g}(\lambda)\right] .
\end{aligned}
$$

In case the operator $H$ has simple spectrum, then Dao-Xeng Xa [13] has shown that $T$ is unitarily equivalent to an operator $S$ acting on $L^{2}\left(E_{H}\right)$ of the form:

$$
S f(s)=s f(s)+i\left[a(s) f(s)+\frac{b(s)}{\pi i} \int_{E_{H}}^{*} \frac{\bar{b}(t) f(t)}{t-s} d t\right] ;
$$

where, $a, b$ are bounded measurable functions on $E_{H}, b(t) \neq 0$ a.e. and $a$ is real valued. The singular integral is interpreted as a Cauchy principal value, that is, $\int^{*}=\lim _{\epsilon \rightarrow 0} \int_{|t-s| \geqslant \epsilon^{\cdot}}$ vector valued generalization of the above result of Dao-Xeng $\mathrm{Xa}$ is in [7]. In fact, the theory of unitary invariants for operators with trace class self-commutator has been significantly developed [3], [6].

In order to have concrete examples of the determining function we observe that the determining function for the operator $S$ defined by (2) is the function

$$
g(x+i y)=\frac{\arg }{\pi}\left[\frac{a(x)+|b(x)|^{2}-y}{a(x)-|b(x)|^{2}-y}\right]
$$

where, $0 \leqslant \arg \leqslant 2 \pi$.

Let $A$ be an operator on $\mathcal{H}$ which has no point spectrum. For $x \in \mathcal{H}$, the function $x(\lambda)=(A-\lambda)^{-1} x$ has a maximal $\mathcal{G}$-valued analytic continuation from the resolvent set of $A$. The domain of this maximal analytic continuation is called the local (analytic) resolvent set of $x$ and is denoted by $\rho_{A}(x)$. The complementary set is called the local spectrum of $x$ and will be denoted by $\sigma_{A}(x)$. If $\sigma$ is a compact subset in the plane, then it is easy to see that $M(\sigma)=\left\{x \in \mathcal{H}: \sigma_{A}(x) \subset \sigma\right\}$ is a linear manifold in $\mathcal{H}$ which is invariant under the operator $A$. Stampfli [12] has shown that $M(\sigma)$ is sometimes a closed subspace when $A$ is hyponormal. More precisely:

THEOREM 2. Let $A$ be a hyponormal operator such that $A^{*}$ has no point spectrum. If $\sigma$ is a compact subset of the plane, then $M(\sigma)$ is a closed subspace invariant under the operator $A$.

Unfortunately, there are hyponormal operators such that $M(\sigma)=\mathscr{H}$ or $M(\sigma)=(0)$ for every compact set $\sigma$. One such example is the unilateral shift. On the other hand there are plenty of examples where the subspace $M(\sigma)$ is a nontrivial invariant subspace for a hyponormal operator (see, e.g. [9] and [12]). Our proof of Theorem 1 will provide more examples where $M(\sigma)$ is nontrivial.

It is interesting to compare the problem of local analytic spectra in the hyponormal case with the cohyponormal case. If $A$ is a cohyponormal operator with empty point spectrum, then there exists a vector $x \in \mathcal{H}$ such that $\sigma_{A}(x)$ is a nonempty proper subset of $\sigma(A)$ [12], however, no analogue of Theorem 2 is known in the cohyponormal case.

If $A$ is an operator on $\mathcal{H}$ which is one-to-one, and $A A^{*}=\int t d G_{t}$ is the spectral resolution of $A A^{*}$, then the vector $x \in \mathcal{H}$ belongs to the domain of 
$A^{-1}$ if and only if $\int t^{-1} d\left(G_{t} x, x\right)<\infty$. In this case the norm of $A^{-1} x$ verifies $\left\|A^{-1} x\right\|^{2}=\int t^{-1} d\left(G_{t} x, x\right)$. Property (v) describes when $\varphi$ is in the domain of $T_{\lambda}^{-1}$ or $\left(\left(T_{\lambda}\right)^{*}\right)^{-1}$.

An irreducible hyponormal operator has no point spectrum. It follows that $\varphi$ is in the domain of $\left(\left(T_{\lambda}\right)^{*}\right)^{-1}$ if and only if $\nu_{g}(\lambda)<\infty$. It should be observed that $\nu_{g}(\lambda)=\infty$ a.e. on the set $\{z: g(z) \neq 0\}$. The fact that the characteristic function of $E_{H} \times E_{J}$ dominates the function $g$ implies that the condition

$$
\int_{E_{H}}|t-p|^{-1} d t<\infty
$$

is sufficient for $\varphi$ to be in the domain of $\left(\left(T_{\lambda}\right)^{*}\right)^{-1}$, for all $\lambda$ such that $\operatorname{Re} \lambda=p$. In fact condition (3) implies $\left\|\left(T_{\lambda}\right)^{*-1} \varphi\right\|$ is bounded on $\operatorname{Re} \lambda=p$.

Let $E$ be a bounded measurable set. Define for real $p$ the function $\nu_{E}(p)=\int_{E}|t-p|^{-1} d t$. This function $\nu_{E}$ is infinite at a.e. point in $E$. On the other hand $\nu_{E}(p)$ is often finite for points $p \notin E$, for example, if $E$ is closed, then $\nu_{E}(p)<\infty$, for $p \notin E$.

There are a couple of examples which are interesting in connection with the function $\nu_{E}$. There is a set $E_{L} \subset I=[0,1]$ of positive measure such that $I \backslash E_{L}$ is a closed nowhere dense set of positive measure and $\nu_{E_{L}}(p)=\infty$, for all $p \in I \backslash E_{L}$. This last example is due to Lusin (see, e.g. [2]). On the other hand, there is another set $E_{0}$ of positive measure in $I$ such that $I \backslash E_{0}$ is a closed nowhere dense set of positive measure with $\nu_{E_{0}}(p)<\infty$, for a.e. $p \in E_{0}$ (see [2]).

The second equality in property (v) implies that $\varphi$ is in the domain of $T_{\lambda}^{-1}$, whenever, $\lambda$ is not in the point spectrum of $T$; moreover, $\left\|T_{\lambda}^{-1} \varphi\right\| \leqslant 1$. This last result has a generalization in Putnam [11].

2. Proof of Theorem 1. We can assume that both operators $T$ and $T^{*}$ have empty point spectrum. It is clear from the discussion in the preceding section that condition (3) implies $\left(\left(T_{\lambda}\right)^{*}\right)^{-1} \varphi$ exists as a bounded $\mathcal{H}$-valued function on $\operatorname{Re} \lambda=p$. An argument similar to that given in Putnam [11] can be used to show that the function $\varphi(\lambda)=\left(T^{*}-\lambda\right)^{-1} \varphi$ is weakly continuous on $\operatorname{Re} \lambda$ $=p$.

Let $C$ be a circle that contains $\sigma\left(T^{*}\right)$ in its interior and let $\gamma$ be the open subinterval of $\operatorname{Re} \lambda=p$ which is contained inside the circle $C$. Form the two closed contours $\Gamma_{1}=\gamma \cup[C \cap[\operatorname{Re} \lambda \geqslant p]]$ and $\Gamma_{2}=\gamma \cup[C \cap[\operatorname{Re} \lambda$ $\leqslant p$ ]]. We assume $\Gamma_{1}, \Gamma_{2}$ are oriented in the counter-clockwise direction. One of the vectors $\varphi_{i}=\int_{\Gamma_{i}} \varphi(\lambda) d \lambda, i=1,2$, is nonzero; here, the integral is defined as a weak integral.

For convenience we assume $\varphi_{1} \neq 0$. It is routine to prove that $\varphi_{1}(\mu)$ $=\int_{\Gamma_{1}} \varphi(\lambda)(\lambda-\mu)^{-1} d \lambda$ is an analytic continuation of $\varphi_{1}(\mu)=\left(T^{*}-\mu\right)^{-1} \varphi_{1}$ onto the domain exterior to $\Gamma_{1}$. This means that $\sigma_{T^{*}}\left(\varphi_{1}\right)$ is a proper subset of $\sigma\left(T^{*}\right)$. It follows from Theorem 2 that $M\left(\sigma_{T^{*}}\left(\varphi_{1}\right)\right)$ is a nontrivial subspace invariant under $T^{*}$. This completes the proof.

3. Conclusion. It is interesting to compare the result in Theorem 1 with the invariant subspace result which follows from property (iv) of the determining function. For definiteness we work with a specific example. Let $E$ be a bounded measurable subset of the real line and consider the singular integral 
operator $T_{E}$ defined on $L^{2}(E)$ by

$$
T_{E} f(s)=s f(s)+\frac{1}{\pi} \int_{E}^{*} \frac{f(t)}{t-s} d t .
$$

Of course, $T_{E}$ is one of the cohyponormal operators defined in equation (2). The principal function is the characteristic function of $E \times[-1,1]$.

The operator $T_{E}$ has an eigenvalue if and only if for some real $p$, the function $\nu_{E^{c}}(p)<\infty$; here, $E^{c}$ is the complement of $E$ relative to some bounded interval containing $E$ in its interior. Theorem 1 gives a nontrivial invariant subspace of $T_{E}$ when $\nu_{E}(p)<\infty$, for some $p$ satisfying ess inf $E<p$ $<$ ess $\sup E$.

The proof of Theorem 1 shows that the operator $T_{F}$ has plenty of nontrivial invariant subspaces whenever $F \subset E_{L}$. It does not give a similar statement about $T_{E_{L}}\left(v_{E_{L}}(p)=\infty\right.$ on $\left.(0,1)\right)$, however, $T_{E_{L}}$ has plenty of eigenvalues.

It was shown above that in the absence of point spectrum of $T$, then $\varphi(\lambda)=(T-\lambda)^{-1} \varphi$ admits a weakly continuous extension (still denoted by $\varphi(\lambda))$ from the resolvent set onto all of $\mathbf{C}$. It develops that $\varphi(\lambda)$ is not analytic at points in the boundary of $\sigma(T)$.

From the fact that $\nu_{g}(\lambda)=\infty$ a.e. on $\{z: g(z) \neq 0\}$, it follows that $\|\varphi(\lambda)\|$ $=1$ for points in every neighborhood of a point $\lambda_{0}$ in the boundary of $\sigma(T)$. On the other hand $\|\varphi(\lambda)\|<1$, for $\lambda \notin \sigma(T)$. This implies that $\varphi(\lambda)$ is not analytic in any neighborhood of $\lambda_{0}$ (see, e.g. Dunford and Schwartz [5, p. 220]).

\section{REFERENCES}

1. R. W. Carey and J. D. Pincus, An invariant for certain operator algebras, Proc. Nat. Acad. Sci. U.S.A. 71 (1974), 1952-1956.

2. _ - Eigenvalues of seminormal operators, examples, Pacific J. Math. 52 (1974), 347-357.

3. Commutators, symbols and determining functions (preprint).

4. K. F. Clancey and C. R. Putnam, The spectra of hyponormal integral operators, Comment. Math. Helv. 46 (1971), 451-456. MR 46 \#31.

5. N. Dunford and J. T. Schwartz, Linear operators. I: General theory, Pure and Appl. Math., vol. 7, Interscience, New York, 1958. MR 22 \#302.

6. J. W. Helton and R. Howe, Integral operators: Commutators, traces, index and homology, Proc. Conf. on Operator Theory, Lecture Notes in Math., vol. 345, Springer-Verlag, Berlin and New York, 1973.

7. J. D. Pincus, Commutators and systems of singular integral equations. I, Acta Math. 121 (1968), 219-249. MR 39 \#2026.

8. , The spectrum of seminormal operators, Proc. Nat. Acad. Sci. U.S.A. 68 (1971), $1684-1685$.

9. C. R. Putnam, Almost isolated spectral parts and invariant subspaces (preprint).

10. Commutation properties of Hilbert space operators and related topics, Ergebnisse der Mathematik und ihrer Grenzgebiete, Band 36, Springer-Verlag, New York, 1967. MR 36 \# 707.

11. - Resolvent vectors, invariant subspaces, and sets of zero capacity, Math. Ann. 205 (1973), 165-171. MR 48 \# 4772.

12. J. G. Stampfli, Spectral subspaces for hyponormal operators (preprint).

13. Dao-Xeng Xa (Tao-hsing Hsia), On non-normal operators. I, Acta Math. Sinica 12 (1962), 216-228 = Chinese Math. 3 (1963), 232-246. MR 26 \#6773.

Institute of Mathematics, ACADEmiei 14, Bucharest, Romania

Department of Mathematics, University of Georgia, Athens, Georgia 30602 